\title{
Sofosbuvir, a Significant Paradigm Change in HCV Treatment
}

\author{
Thomas McQuaid $^{* 1}$, Carolyn Savini ${ }^{2}$ and Star Seyedkazemi ${ }^{3}$ \\ ${ }^{1}$ Medical Affairs, Gilead Sciences, Norwalk, CT, USA; ${ }^{2}$ Medical Affairs, Gilead Sciences, Dallas, TX, USA; ${ }^{3}$ Medical Affairs, Gilead
} Sciences, Foster City, CA, USA

\begin{abstract}
Nucleotide compounds like sofosbuvir, acyclovir, and tenofovir have proven to be amongst the most potent orally available antiviral treatments. These drugs exhibit high efficacy and a wide therapeutic index, with demonstrated utility in a number of chronic viral infections. The approval of Sovaldi ${ }^{\mathrm{TM}}$, brand name for sofosbuvir, by the U.S. Food and Drug Administration heralded improvements in chronic hepatitis C virus (HCV) treatment. Sofosbuvir was originally discovered by Pharmasset Corporation and named PSI7977. It was subsequently acquired and advanced through phase 3 development by Gilead Sciences, Inc. In Sofosbuvir both a unique pharmacology and a high specificity for the HCV ribonucleic acid polymerase are present in a molecule that is well tolerated and highly efficacious. Phase 2 and 3 clinical trials have consistently demonstrated durable and high rates of sustained virologic response (SVR), curing patients in excess of $80 \%$ in all genotypes and $>90 \%$ in treatment-naïve subjects being administered combination therapy with other agents. Harvoni ${ }^{\circledR}$ is the combination of sofosbuvir and the NS5A inhibitor ledipasvir in a fixed-dose oral tablet, and it has demonstrated high SVR rates in patients infected with HCV genotype 1 , without the need for exogenous interferon and/ or ribavirin. Here, we discuss the discovery, development, pharmacologic characterization, and results from the phase 3 trials of sofosbuvir. Hepatitis $C$ is a chronic disease, for which most patients have been undiagnosed, are unwilling to start treatment, or are ineligible for treatment because of the high toxicity and low efficacy of interferon and ribavirin-based therapy. Clinical studies with sofosbuvir have demonstrated significant improvement over the prior standard of care, thus ushering in a new paradigm of HCV treatment and an update of treatment guidelines.
\end{abstract}

Keywords: Ledipasvir; Sofosbuvir; Direct acting antivirals.

Abbreviations: AASLD, American Association for the Study of Liver Diseases; $\mathrm{BCRP}$, breast cancer resistance protein; BMI, body mass index; CYP, cytochrome p450 genes; DAAs, direct acting antivirals; DNA, deoxyribonucleic acid; eGFR, estimated glomerular filtration rate; ESRD, end stage renal disease; FDA, Food and Drug Administration; HCV, hepatitis C virus; HIV, human immunodeficiency virus; IAS-USA, International Antiviral Society-USA; IDSA, Infectious Diseases Society of America; IU, international unit; NS, nonstructural; PegIFN, pegylated interferon; OAT1, organic anion-transporting protein $1 ;$ OCT2, organic cation transporter 2; P-gp, p-glycoprotein; PIs, protease inhibitors; QTc, corrected QT; RAV, resistance-associated variant; RBV, ribavirin; RNA, ribonucleic acid; SVR, sustained virologic response; STR, single-tablet-regimen.

Received: 21 December 2014; Revised: 18 January 2015; Accepted: 19 January 2015

DOI: $10.14218 / \mathrm{JCTH} .2014 .00041$.

*Correspondence to: Thomas McQuaid, Gilead Sciences, 25 Marshall Street Unit 2C, Norwalk, CT 06854, USA. Tel: +1-917-566-3554, Fax: +1-203-274-6713, E-mail: Tom.McQuaid@gilead.com (c) 2015 The Second Affiliated Hospital of Chongqing Medical University. Published by XIA \& HE Publishing Ltd. All rights reserved.

\section{Introduction}

In the last 3 years, hepatitis $\mathrm{C}$ virus ( $\mathrm{HCV}$ ) treatment has undergone significant changes. Direct acting antivirals (DAAs), when combined with pegylated interferon (PegIFN) and ribavirin (RBV), improved rates of cure in chronic HCV infection as compared to treatment with PegIFN and RBV alone. ${ }^{1}$ Protease inhibitors (PIs) were the first class of DAAs to be approved for treatment of $\mathrm{HCV}$ and target the nonstructural protein (NS)3/4A serine protease, which processes the nascent viral poly-protein, allowing for $\mathrm{HCV}$ replication. ${ }^{1}$ The first NS3/4A protease inhibitors approved for use in the U.S., telaprevir and boceprevir, each required coadministration with PegIFN and RBV and the use of complex response-guided therapy algorithms to demonstrate improved sustained virologic response (SVR) rates compared with PegIFN and RBV alone for treatment of HCV genotype $1 .{ }^{1}$ Although this therapeutic approach represented an advancement in the treatment of $\mathrm{HCV}$, it resulted in increased toxicity and increased potential for significant drug-to-drug interactions. $^{1}$

The continued search to improve upon the clinical profile of DAAs led to the evaluation of other key viral targets, including structural and nonstructural proteins. ${ }^{1}$ To date, lead antiviral compounds have been generated against two additional targets. These include the NS5A replication scaffold, also known as the membranous web, and the NS5B ribonucleic acid (RNA)-dependent RNA polymerase (Fig. 1).

The nonstructural protein NS5B, an RNA dependent RNA polymerase, is an attractive target for the development of $\mathrm{HCV}$ therapies as nucleoside and nucleotide polymerase inhibitors have been a mainstay of antiviral therapy for a number of chronic viral diseases. In the HCV lifecycle, replication requires NS5B both to copy the RNA genome and to transcribe messenger RNA. ${ }^{2-4}$ These essential steps in the lifecycle of $\mathrm{HCV}$ are critical for viral replication, and thus inhibition of NS5B prevents viral propagation. ${ }^{4}$

Compounds that inhibit NS5B are classified into two subclasses: nucleos(t)ide inhibitors and non-nucleoside inhibitors. ${ }^{4}$ Nucleos(t)ide inhibitors are analogues of the naturally occurring polymerase substrates and cause premature chain termination when incorporated into the nascent nucleic acid chain. ${ }^{1,4}$ Since the HCV NS5B polymerase's active site is highly conserved across genotypes, nucleos(t)ide inhibitors tend to have similar antiviral activity across all HCV genotypes, referred to as pan-genotypic activity. Many also have a high genetic barrier to the development of drug 


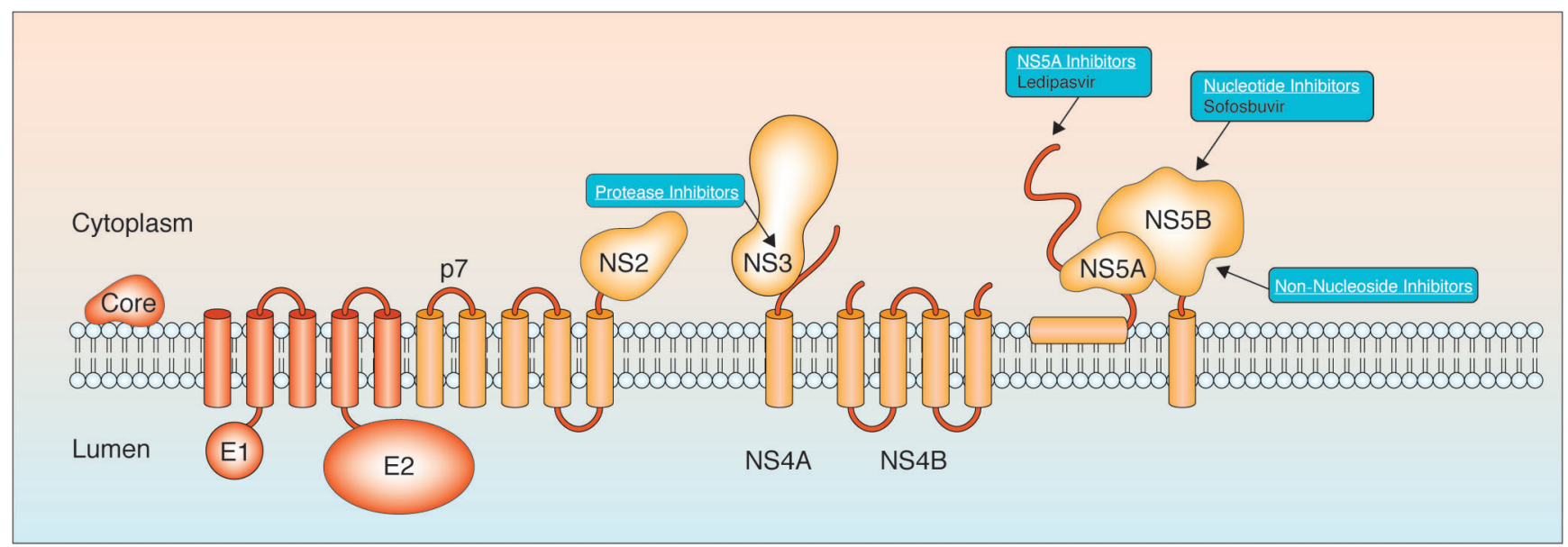

Fig. 1. Overview of direct-acting antivirals (DAAs). DAAs, direct acting antivirals.

resistance. ${ }^{5}$ In contrast, non-nucleoside polymerase inhibitors bind distal to the catalytic site, are less likely to have pan-genotypic activity, and have thus far demonstrated a lower genetic barrier to resistance. ${ }^{5}$

In the quest for an inhibitory NS5B drug prototype, nearly 100 crystal structures of the HCV NS5B were studied. ${ }^{6}$ Visually, the NS5B has a "right hand configuration", with the readily recognized finger, palm, and thumb domains common to many polymerases. ${ }^{6}$ Researchers at Pharmasset Inc. investigated compounds of the nucleoside class that could inhibit HCV replication in multiple HCV genotypes. ${ }^{7}$ Their goal was to identify agents with improved potency and enhanced pharmacokinetic properties, thereby allowing for once-daily dosing at low doses that could achieve high concentrations of the active triphosphate in the liver. ${ }^{7,8}$

\section{Discovery and development}

The goal during development of sofosbuvir was to create a prodrug compound with optimal oral absorption and potent antiviral activity. ${ }^{8}$ Following absorption, liver enzymes hydrolyze the terminal carboxylic acid ester of the phosphoramidite moiety, and a cascade of chemical and enzymatic events produce the pharmacologically active uridine triphosphate analogue. The triphosphate of 2 '-alpha-F-2'-beta-C-methyl uridine is a potent inhibitor of the NS5B polymerase and exhibits a long intracellular half-life of $36 \mathrm{~h} .{ }^{9}$ Initially the antiviral potency of a mixture of PSI-7977 diastereomers was evaluated, but it was later discovered that a purified nonracemic single diastereomer produced significantly greater reductions in HCV RNA. ${ }^{8}$ The discovery and characterization of this single diastereomer resulted in the first crystallization and X-ray derived structure of a nucleotide phosphoramidiate, a DAA with improved potency and enhanced pharmacokinetic properties. ${ }^{7,8}$ This compound, PSI-7977, is now known as sofosbuvir and allows for once-daily dosing and the potential for generating high concentrations of the active triphosphate in the liver.7,8 Gilead Sciences acquired Pharmasset Inc. and PSI-7977 in November of 2011 and conducted additional preclinical and clinical research to evaluate the pharmacology and clinical safety and efficacy (Phase $2 b$ and 3 ) of sofosbuvir, the results of which are summarized here. On December 6, 2013 the Food and Drug
Administration (FDA) approved Sovaldi ${ }^{\mathrm{TM}}$ (sofosbuvir) $400 \mathrm{mg}$ tablets for use in combination with other agents for the treatment of chronic HCV infection in adults.

\section{Pharmacology}

\section{Absorption, metabolism, and elimination}

Sofosbuvir is a prodrug administered once-daily as a $400 \mathrm{mg}$ oral tablet without a food or fasting requirement. ${ }^{10}$ Sofosbuvir is absorbed with a peak plasma concentration observed at $\sim 0.5-2 \mathrm{~h}$ post-dose. ${ }^{11}$ Following absorption, sofosbuvir is metabolized in hepatocytes, where it is converted to the active nucleoside triphosphate form, GS461203. ${ }^{12}$ This requires two phosphorylation events that are mediated by endogenous host cell kinases. ${ }^{12}$ GS-461203 has a long half-life of approximately $18 \mathrm{~h}$, supportive of once daily dosing. ${ }^{12}$ GS-461203 is irreversibly metabolized to the inactive phosphate free metabolite of the nucleotide, GS$331007 .{ }^{12}$ This metabolite is largely eliminated by passive filtration in the renal glomerulus. ${ }^{12}$ Prodrug not taken up by cells is also converted to GS-331007 (Fig. 2). ${ }^{12}$ GS-331007 from intracellular and extracellular metabolism accounts for greater than $90 \%$ of the systemically circulating drug-related material, while the parent sofosbuvir molecule accounts for approximately $4 \%$ of circulating drug related material and has a relatively short half-life of $0.5-0.8 \mathrm{~h} .{ }^{10,13}$ Mass balance study of sofosbuvir elucidated that $76 \%$ of the drug was eliminated in the urine primarily as the GS331007 metabolite, $14 \%$ in feces, and $3 \%$ in expelled air. ${ }^{11}$ While GS-331007 is eliminated through the kidneys both by passive and active filtration/transport, it is not a substrate for the renal transporters (organic anion-transporting peptide 1 (OAT1), organic cation transporter 2 (OCT2), MATE1, p-glycoprotein $(\mathrm{P}-\mathrm{gp})$, and breast cancer resistance protein (BCRP)) in vitro. ${ }^{13}$

\section{Drug-to-drug interactions}

Sofosbuvir is a substrate of the drug transporters P-gp and BCRP, while GS-331007 is not. As such, sofosbuvir should not be administered with potent inducers of intestinal P-gp, such as rifampin and Saint John's wort, which may result in reduced 


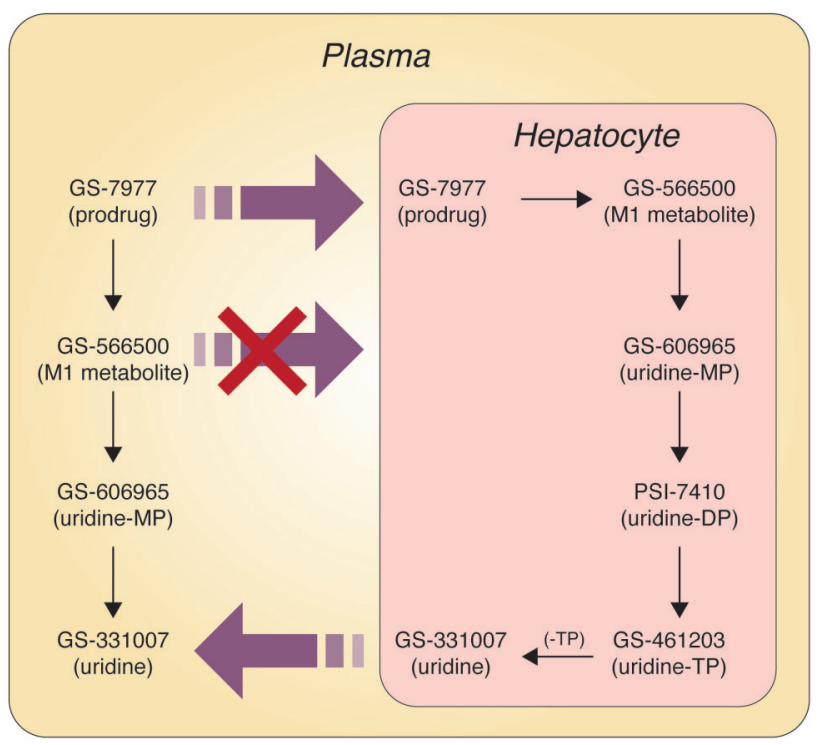

Fig. 2. Sofosbuvir intracellular activation.

absorption (see Table 1). ${ }^{13,14}$ Additionally, co-administration of sofosbuvir with select anticonvulsants, rifabutin, rifapentine, or tipranavir/ritonavir, is not recommended. ${ }^{11}$

The cytochrome p450 genes (CYP3A) encode a number of monooxygenase enzymes that are used to metabolize many drugs. Since sofosbuvir is not metabolized by, nor does it inhibit or induce CYP3A, it does not have many of the drug-todrug interactions involved with other HCV antivirals. ${ }^{11}$ Sofosbuvir has been evaluated and found to have no clinically significant drug interactions with many of the common medications metabolized by CYP3A enzymes, including tacrolimus, cyclosporine, and methadone. ${ }^{11}$

It is estimated that up to $30 \%$ of human immunodeficiency virus (HIV) positive patients are infected with $\mathrm{HCV}$, and thus drug-to-drug interactions with HIV antiretrovirals can be problematic in HCV treatment. The fixed-dose combination emtricitabine/tenofovir disoproxyl fumarate, the single-tablet regimens of emtricitabine/rilpivirine/tenofovir disoproxyl fumarate and efavirenz/emtricitabine/tenofovir disoproxyl fumarate, ritonavir-boosted HIV protease inhibitors darunavir and Atazanavir, and raltegravir are among the commonly used antiretrovirals evaluated in drug interaction studies. These combination were found to have no clinically significant interaction with sofosbuvir. ${ }^{11}$

\section{Evaluation in hepatic and renal impairment}

Availability of safe treatment options for those with advanced fibrosis and cirrhosis, as well as those with diminished renal function, is a significant medical need for patients with HCV. The pharmacokinetics, safety, and antiviral activity of sofosbuvir were evaluated in HCV-infected subjects with moderate or severe hepatic impairment. ${ }^{15}$ Sofosbuvir was well tolerated during the 7 day dosing period. ${ }^{15}$ Systemic exposure to sofosbuvir was approximately two-fold higher in cirrhotic subjects relative to $\mathrm{HCV}$-infected non-cirrhotic subjects, but there was no change in exposure to GS-331007. ${ }^{15}$ Additionally, HCV RNA was significantly decreased in subjects with Child Pugh Class B and C, where respective median day
Table 1. Impact of intestinal P-gp inducers on sofosbuvir (SOF)

\begin{tabular}{ll}
\hline $\begin{array}{l}\text { Concomitant drug class: } \\
\text { drug name }\end{array}$ & Effect on concentration \\
\hline Anticonvulsants: & $\downarrow$ SOF \\
Carbamazepine & \\
Phenytoin & \\
Phenobarbital & \\
Oxcarbazepine & \\
Antimycobacterials: & $\downarrow$ SOF \\
Rifabutin & \\
Rifapentine & \\
Rifampin & \\
Antiretrovirals: & \\
Tipranavir/ritonavir & \\
Herbal Supplements: & $\downarrow$ SOF \\
St. John's Wort & \\
\hline
\end{tabular}

seven change from baseline in HCV RNA was -3.65 and $-3.14 \log _{10}$ international unit $(\mathrm{IU}) / \mathrm{mL}$, respectively. These observed declines were less profound than in subjects without cirrhosis. ${ }^{15}$ Based on these data, no dose adjustment of sofosbuvir is warranted in mild, moderate, or severe hepatic impairment, although viral suppression may be slower among subjects with Child-Pugh Class B and C.

As in patients with advanced hepatic disease, patients with renal failure present challenges to the treatment of $\mathrm{HCV}$ with historically approved regimens. The pharmacokinetics of sofosbuvir and GS-331007 were evaluated in HCV-negative subjects with normal renal function and mild, moderate, or severe renal impairment. ${ }^{16}$ Subjects with mild, moderate, and severe renal impairment had approximately 56\%, 90\%, and $456 \%$ higher GS-331007 AUC, respectively, than subjects with normal renal function. ${ }^{16}$ The pharmacokinetics of sofosbuvir and GS-331007 were also evaluated in subjects with end stage renal disease (ESRD), dosed predialysis and postdialysis. ${ }^{16}$ In subjects with ESRD, dialysis is essential to eliminate GS-331007 from the body. ${ }^{16}$ Based on these evaluations, no dose adjustment of sofosbuvir is warranted in mild or moderate renal impairment, when estimated glomerular filtration rate (eGFR) is $\geq 30 \mathrm{~mL} / \mathrm{min} / 1.73 \mathrm{~m}^{2}$. The safety and efficacy of sofosbuvir has not been established in patients with severe renal impairment (eGFR $<30 \mathrm{~mL} / \mathrm{min}$ ) and ESRD, including patients requiring hemodialysis, and therefore, no dose recommendation can be made at this time. Studies are currently underway to determine dosing and effectiveness of sofosbuvir in these patients.

\section{Toxicology studies}

Nucleos(t)ide analogues that have high affinity for mitochondrial polymerases can cause mitochondrial damage, and ultimately, cell death. This may result in many of the toxicities that have been seen with other members of this antiviral class. Sofosbuvir was found in vitro to have a low affinity for host cellular and mitochondrial deoxyribonucleic acid (DNA) and RNA polymerases, contributing to a low overall toxicity profile and differentiating sofosbuvir from other nucleos(t)ide analogue antivirals (Table 2). ${ }^{13}$ The effect of sofosbuvir 400 and $1200 \mathrm{mg}$ on corrected QT (QTc) interval was also evaluated in a randomized, single-dose, placebo-, and active-controlled (moxifloxacin $400 \mathrm{mg}$ ) thorough QT study in 59 healthy subjects. ${ }^{17}$ The maximum change in 
time-matched and baseline-adjusted QTcF intervals following a single dose of sofosbuvir (400 and $1200 \mathrm{mg}$ ) and moxifloxacin (400 mg; positive control) were 2.36, 2.57, and 11.3 , respectively. ${ }^{17}$ Sofosbuvir administered up to three times the maximum recommended dose $(1200 \mathrm{mg})$ did not have a clinically meaningful effect on QTcF intervals. ${ }^{17}$

\section{Clinical trials}

Sofosbuvir is indicated in the U.S. for treatment of chronic HCV genotypes 1 through 4 in treatment-naïve and experienced patients, with or without HIV co-infection when used in combination with other antiviral agents. ${ }^{11}$ These indications were granted by the U.S. FDA after a review of data from phase 2 and 3 clinical trials. These trials evaluated the safety and efficacy of 12 to 24 weeks of sofosbuvir in combination with RBV, with and without PegIFN for treatment of genotypes 1 through $4 .{ }^{11}$ The recommended regimens and treatment duration for sofosbuvir combination therapy in HCV mono-infected and HCV/HIV1 co-infected patients are summarized in Table 3.

\section{Phase 3 studies in genotypes 1, 4, 5 and 6}

The phase 3 study, NEUTRINO, evaluated 327 treatment-naïve subjects infected with chronic HCV genotypes 1, 4, 5, and 6, including 54 patients $(17 \%)$ with compensated cirrhosis. ${ }^{18}$ Subjects were treated with 12 weeks of sofosbuvir $400 \mathrm{mg}$ once daily, weight-based dosed RBV, $1200 \mathrm{mg} \geq 75 \mathrm{~kg}$ and $1000 \mathrm{mg}<75 \mathrm{~kg}$ (Ribasphere ${ }^{\circledR}$ ) divided in two doses, and pegIFN once weekly (Pegasys ${ }^{\circledR}$ ).${ }^{18}$ Ninety-nine percent of subjects achieved HCV RNA below the limit of quantification $(<25 \mathrm{IU} / \mathrm{mL})$ by week four and end of treatment, and $90 \%$ achieved SVR12. ${ }^{18}$ On-treatment viral kinetics did not predict SVR, thus, response-guided therapy is not indicated with sofosbuvir-based therapy. ${ }^{18}$ Subjects with genotype 1 achieved $90 \%$ SVR 12 , while $96 \%$ of genotype 4 and $100 \%$ of genotypes 5 and 6 subjects achieved SVR. ${ }^{18}$ Subjects with cirrhosis, the majority of whom were infected with genotype 1 , had an overall SVR12 rate of $80 \% .{ }^{18}$ High rates of SVR12 were seen across all genotypes and in patients with factors that have historically predicted negative outcomes (high body mass index (BMI), black race, IL-28B non-CC genotype, and high HCV viral load). ${ }^{18}$

No subject experienced on-treatment virologic breakthrough, and relapse accounted for all virologic failures. ${ }^{18}$ Deep virologic sequencing analysis of samples from subjects who relapsed did not identify the sofosbuvir resistanceassociated variant (RAV), S282T. ${ }^{18}$ The combination of sofosbuvir, RBV, and PegIFN was safe and well tolerated, with fewer than $2 \%$ of subjects discontinuing therapy. ${ }^{18}$ The most commonly reported adverse events were fatigue, headache, insomnia, and nausea. ${ }^{18}$ These side effects are typically associated with RBV and/or PegIFN. The addition of sofosbuvir to this combination did not increase the severity of reported adverse events. ${ }^{18}$

Based on results observed in NEUTRINO, the U.S. FDA modeled an SVR rate in HCV genotype 1 patients who had failed prior PegIFN-based therapy. ${ }^{11}$ The FDA model predicted that the response rate of treatment-experienced patients treated with 12 weeks of sofosbuvir, PegIFN, and RBV would approximate the observed response rate in NEUTRINO subjects with multiple baseline factors traditionally associated with a lower response to interferon-based treatment. ${ }^{11}$ These factors include genotype $1 \mathrm{HCV}$, Metavir F3/F4 stages of fibrosis, nonCC should be hyphenated: non-CC IL-28B haplotype, and $\mathrm{HCV}$ RNA $>800,000 \mathrm{IU} / \mathrm{mL}$. The SVR12 rate in NEUTRINO among the subset of 52 subjects with all four of these characteristics was $71 \%(37 / 52) .{ }^{11}$

The safety and efficacy of 24 weeks of the interferon-free regimen of sofosbuvir plus RBV for treatment of genotype 1 $\mathrm{HCV}$ was evaluated in a phase 2 single-center, two part, randomized controlled trial conducted at the Clinical Research Center of the National Institutes of Health. ${ }^{19}$ The SPARE study enrolled 60 subjects, whose baseline characteristics reflected a traditionally difficult to cure population, including black race $(83 \%), B M I>30 \mathrm{~kg} / \mathrm{m}^{2}(48 \%)$, non-CC IL-28B haplotype $(81 \%)$, genotype 1 a $(70 \%)$, advanced liver disease $(23 \%)$, and baseline HCV RNA levels $>800,000 \mathrm{IU} / \mathrm{mL}(62 \%) .{ }^{19}$ Overall, $68 \%$ of genotype 1 subjects treated with sofosbuvir and weight-based dose of RBV achieved SVR. ${ }^{19}$

\section{Phase 3 studies in genotypes 2 and 3}

The phase three clinical trials evaluating genotypes 2 and 3 included FISSION, POSITRON, FUSION, and VALENCE. These trials evaluated the safety and efficacy of different durations of therapy using all oral regimens of sofosbuvir and weightbased RBV in genotypes 2 and $3 \mathrm{HCV}$ infection.

The FISSION trial randomized 499 subjects, including 100 patients with cirrhosis, to receive either sofosbuvir $(400 \mathrm{mg}$ once daily) and weight-based RBV for 12 weeks or to receive PegIFN alfa-2a and RBV (800 mg daily divided in two doses) for 24 weeks. ${ }^{18}$ The primary endpoint was SVR12, with a prespecified noninferiority margin of $15 \% .{ }^{18}$ Baseline demographics were well balanced between the study arms. ${ }^{18}$ The majority of subjects in both arms were Caucasian, which is representative of the demographics of patients infected with genotypes 2 and $3 .{ }^{18}$ The majority of the subjects in this study were infected with HCV genotype 3, comprising $73 \%$ of the subjects enrolled. ${ }^{18}$ Overall, $67 \%$ of subjects in the PegIFN + RBV arm and $67 \%$ of the subjects in the sofosbuvir + RBV arm achieved SVR12; therefore, the study met its primary endpoint of noninferiority. ${ }^{18}$ SVR occurred in $97 \%$ of the HCV genotype 2 subjects and in $56 \%$ of HCV genotype 3 subjects treated with

Table 2. Inhibition of host polymerase

\begin{tabular}{llllll}
\hline & \multicolumn{5}{c}{ IC50 $\mu \mathrm{M}$} \\
\hline & DNA Pol $\alpha$ & DNA Pol $\beta$ & DNA Pol $\gamma$ & RNA Pol II & mtRNAP \\
\hline SOF Triphosphate & $>200$ & $>200$ & $>200$ & $>200$ & $>500$ \\
Controls & aphidicolin & $3^{\prime}$-dTTP & $3^{\prime}$-dTTP & $\alpha$-amanitin & $3^{\prime}$-dGTP \\
IC 50 & $(7.3)$ & $(1.4)$ & $(0.74)$ & $(0.0024)$ & $(1.9)$ \\
\hline
\end{tabular}

The triphosphate of sofosbuvir (SOF) did not inhibit human DNA or RNA polymerases. 
McQuaid T. et al: Sofosbuvir in HCV treatment

Table 3. Recommended sofosbuvir combination regimen and treatment duration

\begin{tabular}{|c|c|c|}
\hline & Treatment & Duration \\
\hline Patients with genotype 1 or 4 & $\begin{array}{l}\text { Sofosbuvir } 400 \mathrm{mg} \text { once daily, RBV } 1000 / 1200 \text { in two divided } \\
{\text { dose, }{ }^{a} \text { and PegIFN once weekly }}^{b}\end{array}$ & 12 weeks \\
\hline IFN-ineligible patients with genotype 1 & $\begin{array}{l}\text { Sofosbuvir } 400 \text { mg once daily and RBV 1000/1200 in two } \\
\text { divided dose }\end{array}$ & 24 weeks \\
\hline Patients with genotype 2 & $\begin{array}{l}\text { Sofosbuvir } 400 \mathrm{mg} \text { once daily and RBV 1000/1200 in two } \\
\text { divided dose }\end{array}$ & 12 weeks \\
\hline Patients with genotype 3 & $\begin{array}{l}\text { Sofosbuvir } 400 \text { mg once daily and RBV } 1000 / 1200 \text { in two } \\
\text { divided dose }\end{array}$ & 24 weeks \\
\hline
\end{tabular}

${ }^{\mathrm{a}}$ Dose of ribavirin is weight-based $(<75 \mathrm{~kg}=1000 \mathrm{mg}$ and $\geq 75 \mathrm{~kg}=1200 \mathrm{mg}$ ).

${ }^{\mathrm{b}}$ Dose of PegIFN alfa is per the prescribing information.

sofosbuvir and RBV. In comparison, the response rates were $78 \%$ and $63 \%$ in subjects treated with PegIFN and RBV respectively. ${ }^{18}$ Genotype 2 and 3 subjects with cirrhosis who received sofosbuvir and RBV achieved SVR rates of $91 \%$ and $34 \%$, respectively. ${ }^{18}$ SVR rates for genotype 2 and 3 subjects who received PegIFN and RBV were $62 \%$ and $30 \%$, respectively. ${ }^{18}$ The difference in response rates with sofosbuvir and RBV vs. PegIFN and RBV was statistically significant $(p<0.001)$ among genotype 2 infected subjects. ${ }^{18}$ Overall virologic relapse occurred among $30 \%$ of subjects in the sofosbuvir and RBV arm and among $22 \%$ of subjects in the PegIFN and RBV arm. ${ }^{18}$ No S282T RAVs were identified upon deep sequencing of virus samples from subjects who did not achieve SVR. ${ }^{18}$ Sofosbuvir combined with RBV was well tolerated, and discontinuation of treatment occurred rarely at $1 \%$, compared to $11 \%$ in the PegIFN and RBV arm. ${ }^{18}$ None of the discontinuations due to adverse events in the sofosbuvircontaining arm was deemed related to sofosbuvir. ${ }^{18}$

POSITRON was a randomized, double-blinded, placebocontrolled trial that evaluated the safety and efficacy of 12 weeks of treatment with sofosbuvir and weight-based RBV in 207 subjects compared to 71 subjects who received placebo. ${ }^{20}$ Subjects enrolled in this trial were intolerant to, ineligible for, or unwilling to receive interferon-based therapy. Sixteen percent of subjects had evidence of cirrhosis at baseline. ${ }^{20}$ Both study arms were balanced with respect to subject demographics and baseline characteristics, including 51\% genotype 2 and $49 \%$ genotype $3 .^{20}$ None of the subjects who received placebo achieved SVR, while $93 \%$ of genotype 2 and $61 \%$ of genotype 3 subjects who received active treatment achieved SVR ( $p$ $<0.001) .{ }^{20}$ Robust response was seen in both HCV Genotypes 2 and 3 subjects without cirrhosis, with $81 \%$ achieving SVR ( $92 \%$ of subjects with HCV genotype 2 and $68 \%$ of those with HCV genotype 3 ). ${ }^{20}$ Cirrhosis did not negatively impact outcomes among genotype 2 subjects, $94 \%$ of whom achieved SVR. ${ }^{20}$ However, genotype 3 subjects with cirrhosis had a blunted response with an SVR rate of $21 \% .{ }^{20}$ Failures to achieve SVR were due to relapse, and no S282T RAVs were identified upon deep sequencing of samples from subjects who did not achieve SVR. ${ }^{20}$ Treatment with sofosbuvir was well tolerated, with reported adverse events being those most commonly associated with RBV, such as fatigue, headache, nausea, insomnia, and rash. ${ }^{20}$ Study discontinuation due to adverse events was minimal at $2 \%$ in the sofosbuvir arm and $4 \%$ in the placebo arm. ${ }^{20}$

FUSION was a randomized, double-blinded trial that evaluated the safety and efficacy of either 12 or 16 weeks of treatment with sofosbuvir and weight-based RBV in 201 subjects who did not achieve SVR with prior interferon-based treatment (relapsers and nonresponders). ${ }^{20}$ Baseline subject demographics and disease characteristics were balanced between the 12 and 16 week arms. ${ }^{20}$ Sixty-three percent of subjects were infected with genotype $3 \mathrm{HCV}$, and $34 \%$ of the overall population had evidence of cirrhosis at baseline. ${ }^{20}$ The 12 week duration of therapy resulted in $50 \%$ of subjects achieving an SVR12, compared to $71 \%$ with 16 weeks of therapy. ${ }^{20}$ A substantial increase in SVR rate was seen in genotype 3 subjects who received 16 weeks of therapy. The SVR12 results in genotype 3 subjects treated for 12 weeks was $30 \%$ compared to $62 \%$ in subjects who received 16 weeks of treatment. ${ }^{20}$ All virologic failures were due to relapse, and no S282T RAVs were identified upon deep sequencing. ${ }^{20}$ Treatment discontinuations were infrequent, occurring at $1 \%$ in the 12 week arm compared to $0 \%$ in the 16 week study arm. ${ }^{20}$ Sofosbuvir-based therapy was well tolerated, with the most common adverse events being those attributed to RBV. ${ }^{20}$ No additional safety signals were identified, and adverse events were not increased with the additional 4 weeks of therapy. ${ }^{20}$

Results of these trials demonstrated that 12 weeks of sofosbuvir plus RBV was optimal for genotype 2 patients, whereas genotype 3 patients would benefit from extending treatment duration. ${ }^{20}$ The VALENCE trial was conducted to confirm the treatment duration for genotype 2 infected patients and to explore the efficacy of extending treatment duration to 24 weeks for genotype 3 infected patients.

The VALENCE trial evaluated the efficacy and safety of sofosbuvir and weight-based RBV in 419 HCV treatmentnaïve and experienced genotype 2 and 3 infected subjects. ${ }^{21}$ HCV genotype 2 subjects received 12 weeks of therapy, while genotype 3 subjects were treated for 24 weeks. ${ }^{21}$ SVR 12 was achieved in $93 \%$ of HCV genotype 2 subjects and in $84 \%$ of subjects with genotype $3 .^{21}$ The extension of therapy to 24 weeks in genotype 3 subjects substantially improved SVR rates, which defined the recommended duration of therapy of 24 weeks for genotype 3 infection. ${ }^{21}$ Failures to achieve SVR were due to relapse, and no S282T RAVs were identified upon deep sequencing. ${ }^{21}$ The extension of therapy to 24 weeks in genotype 3 subjects did not demonstrate a significant increase in adverse events or discontinuations. ${ }^{21}$

The sofosbuvir pivotal trials across all genotypes demonstrated SVR12 rates greater than $90 \%$ in treatment-naïve subjects (Fig. 3).

\section{Clinical trials in HCV/HIV co-infection}

PHOTON-1 evaluated the safety and efficacy of 12 or 24 weeks of an interferon-free all-oral regimen of sofosbuvir $400 \mathrm{mg}$ daily and weight-based RBV dosed twice daily in 
HCV/HIV co-infected subjects. ${ }^{22}$ This study enrolled 223 $\mathrm{HCV} / \mathrm{HIV}$ co-infected subjects, including treatment-naïve genotype 1 and both treatment-naïve and experienced genotype 2 and 3 subjects. ${ }^{22}$ Subjects infected with genotype 1 were treated for 24 weeks, treatment-naïve genotype 2 and 3 subjects were treated for 12 weeks, and treatmentexperienced genotype 2 and 3 subjects were treated for 24 weeks duration. ${ }^{22}$ In the study, greater than $90 \%$ of subjects were receiving concomitant antiretroviral medications for the treatment of HIV-1. ${ }^{22}$ Treatment-naïve genotype 1, 2, and 3 subjects achieved SVR12 rates of $76 \%, 88 \%$, and $67 \%$, respectively (Fig. 4). ${ }^{22}$ Ninety-two percent and $94 \%$ of treatment-experienced genotype 2 and 3 subjects achieved SVR12, respectively. ${ }^{22}$ One HCV genotype 1 subject and one HCV genotype 2 subject experienced virologic breakthrough in this study. ${ }^{22}$ Notably, both subjects were found to have undetectable plasma drug concentrations, consistent with nonadherence. ${ }^{22}$ Relapse accounted for all other subjects that did not achieve SVR, and no S282T RAVs were identified by deep sequencing. ${ }^{22}$ Sofosbuvir and RBV were safe and well tolerated with fewer than $4 \%$ of subjects discontinuing therapy due to an adverse event. ${ }^{22}$ The most common adverse events were fatigue, headache, insomnia, and nausea, consistent with those that have been associated with RBV in previous studies. ${ }^{22}$ Additionally, HCV treatment with sofosbuvir and RBV had no impact on CD4 percentage or HIV viral load. ${ }^{22}$ Sofosbuvir was also evaluated in an additional HCV/HIV co-infection study that was conducted at a single site in Puerto Rico. ${ }^{23}$ This study evaluated the safety and efficacy of 12 weeks of sofosbuvir combined with PegIFN and RBV for treatment-naïve, genotypes 1-4 HCV/ HIV co-infected subjects without cirrhosis. ${ }^{23}$ Twenty-one of twenty-three subjects (91\%) achieved SVR12. ${ }^{23}$

Efficacy and safety data from both of these studies in HCV/ HIV co-infection were consistent with previously reported results in HCV mono-infected subjects (Fig. 4). Furthermore, given the high rates of response observed in the PHOTON-1 study, the labeling includes an indication for the use of sofosbuvir and RBV for 24 weeks in HCV genotype 1 patients who are interferon ineligible.

The American Association for the Study of Liver Diseases (AASLD) and the Infectious Diseases Society of America
(IDSA), in collaboration with the International Antiviral Society-USA (IAS-USA), announced the launch of a new website, HCVguidelines.org in January 2014. In contrast with traditional treatment guidelines published in Hepatology, this web-based guidance will be revised regularly with new therapeutic options as they receive FDA approval.

\section{Sofosbuvir-based all-oral DAA therapy}

The overarching goal of research in the HCV arena has been to develop highly efficacious, oral, and safe DAAs or combinations of DAAs that can be used without interferon, and possibly, without RBV. Sofosbuvir has been studied in combination with ledipasvir, an NS5A inhibitor, as a singletablet-regimen (STR) in phase 3 clinical trials for patients with HCV genotype 1 infection. Additionally, phase 2 trials have examined safety and efficacy of this STR in other genotypes. STRs are a mainstay of HIV treatment and demonstrate increased adherence and reduced resistance as compared to multitablet regimens. On October 10, 2014 the FDA approved Harvoni $^{\circledR}$ (ledipasvir/sofosbuvir), the first once daily STR for treatment of chronic HCV in genotype 1 infected patients, including patients with compensated cirrhosis. ${ }^{24}$ The recommended treatment duration for ledipasvir/sofosbuvir STR in patients with chronic HCV genotype 1 infection is summarized in Table 4.

The safety and efficacy of once-daily ledipasvir/sofosbuvir was evaluated in three phase 3 studies, conducted in 1,952 HCV genotype-1 infected subjects. ${ }^{25-27}$ The study population included $308(16 \%)$ black patients, $224(16 \%)$ compensated cirrhotics, $591(26 \%)$ patients with BMI $\geq 30 \mathrm{~kg} / \mathrm{m}^{2}$, and $1,597$ (82\%) with high HCV RNA ( $\geq 800,000 \mathrm{IU} / \mathrm{mL}){ }^{25-27}$ ION-1 is an open-label phase 3 study that evaluated the safety and efficacy of ledipasvir/sofosbuvir in $865 \mathrm{HCV}$ genotype 1 subjects with and without cirrhosis. ${ }^{26}$ Subjects were randomized $1: 1: 1: 1$ to receive 12 or 24 weeks of ledipasvir/sofosbuvir with or without weight-based dose RBV. ${ }^{26}$ High rates of SVR12 (98-99\%) were achieved across all arms, indicating that a 12 week duration of ledipasvir/ sofosbuvir STR was as efficacious as a 24 week duration and that RBV did not increase SVR (Fig. 5). ${ }^{26}$ Given the high rates of SVR observed in phase 2 studies among genotype 1

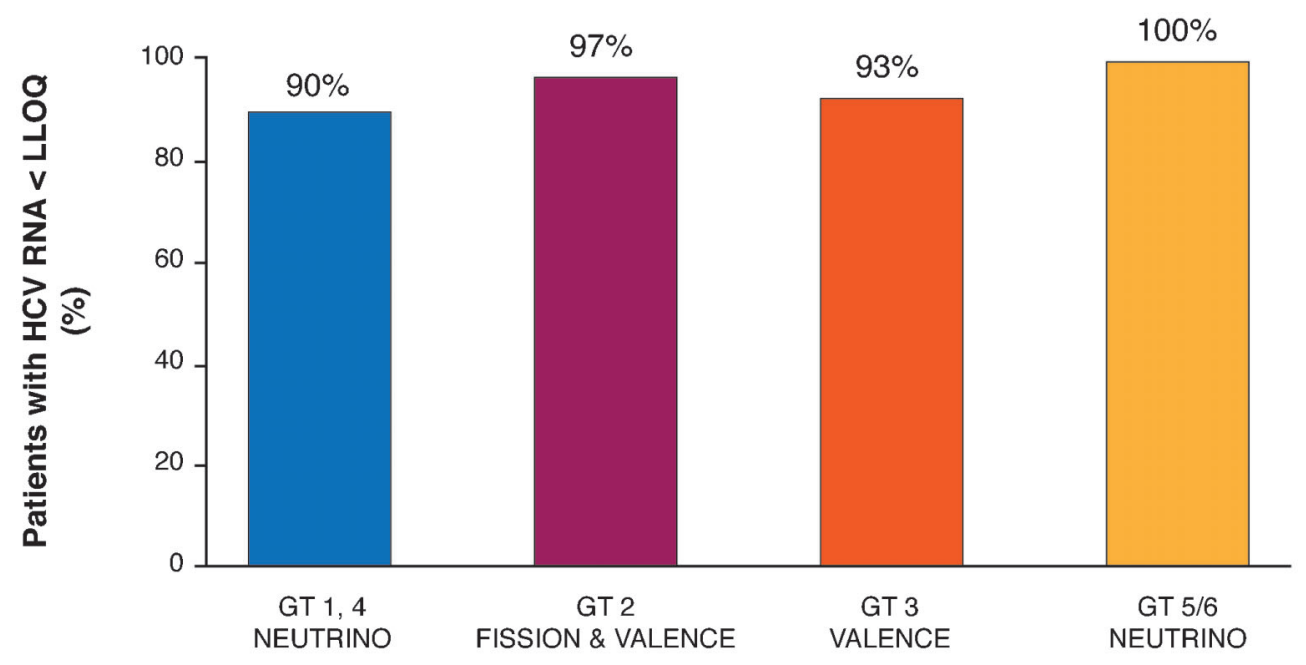

Fig. 3. SVR 12 across treatment-naïve genotypes 1, 2, 3, 4, 5, 6. 
McQuaid T. et al: Sofosbuvir in HCV treatment

$$
\begin{gathered}
\text { GT } 1 \\
\text { SOF+RBV+PegIFN } \\
12 \text { weeks }
\end{gathered}
$$

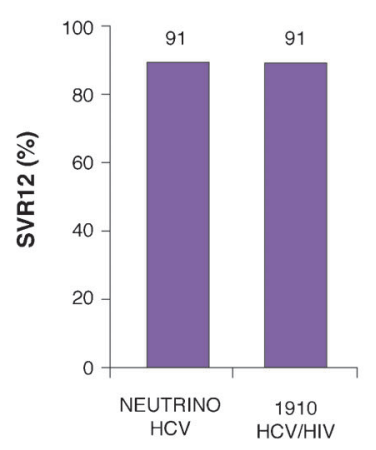

GT 1

$\mathrm{SOF}+\mathrm{RBV}$

24 weeks

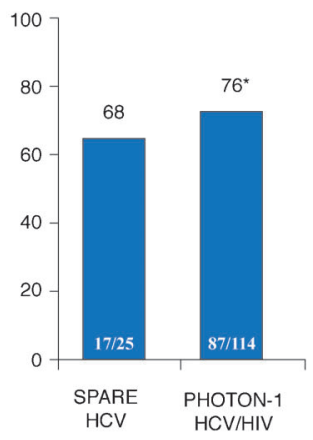

GT 2

$\mathrm{SOF}+\mathrm{RBV}$

12 weeks

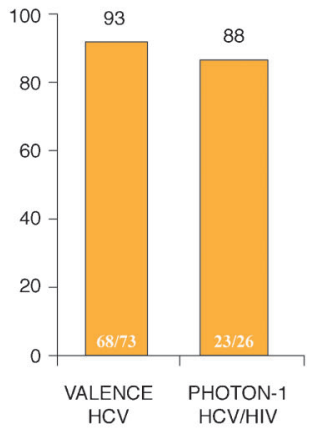

GT 3
SOF+RBV

12 weeks

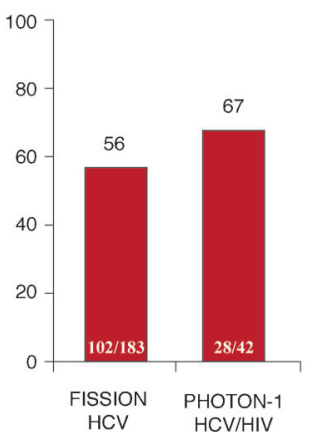

GT 3

SOF+RBV

24 weeks

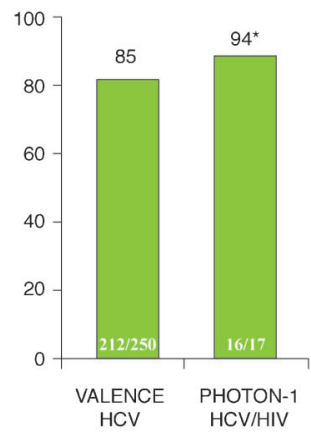

Fig. 4. SVR12 in HCV mono-infected and HCV/HIV co-infected sofosbuvir (SOF) + RBV \pm PegIFN x 12 or 24 weeks. Similar response rates in HCV/HIV coinfected patients compared to HCV mono-infected patients.

subjects without cirrhosis treated with ledipasvir/sofosbuvir for 8 and 12 weeks with or without RBV, the feasibility of shortening treatment duration was evaluated in the phase 3 randomized, open-label ION-3 study. ${ }^{27}$ This study compared the safety and efficacy of 8 weeks of the ledipasvir/sofosbuvir STR with and without RBV to a 12 week course of the STR in 647 genotype 1 treatment-naïve subjects without cirrhosis. ${ }^{27}$ SVR12 ranged from $93-96 \%$ across all arms, establishing the noninferiority of the 8-week duration of therapy with the ledipasvir/sofosbuvir STR without RBV to the other treatment arms (Fig. 5). ${ }^{27}$ In a posthoc analysis of SVR12, having 6 million IU/mL of HCV RNA or greater at baseline was associated with higher relapse in the 8 week arms of the study. ${ }^{24}$ To evaluate the safety and efficacy of this STR in treatment-experienced genotype 1 patients, ION-2 randomized 440 subjects, with and without cirrhosis, to receive 12 versus 24 weeks of ledipasvir/sofosbuvir with and without weight-based RBV. ${ }^{25}$ Two hundred and thirty-one (53\%) of subjects had previously failed to respond to treatment with an HCV NS3/4A protease-inhibitor combined with PegIFN and RBV. $^{25}$ SVR12 was achieved by $94-99 \%$ of subjects across all arms, and addition of RBV did not increase SVR (Fig. 5). ${ }^{25}$ The most common adverse events in the ION studies were fatigue, headache, nausea, and insomnia. ${ }^{25}$ These adverse events, in addition to laboratory abnormalities, occurred more frequently in the RBV-containing arms of the studies. ${ }^{25}$

\section{Conclusions}

The last 5 years have seen significant advances in the treatment of chronic HCV with improvements in efficacy as well as safety and tolerability. Sofosbuvir, a nucleotide analogue, represents a first in class antiviral with unique safety and efficacy properties. These include convenient dosing, limited drug-to-drug interactions, high efficacy, a high barrier to genetic resistance, and high rates of SVR when used with other currently approved HCV antiviral agents. In clinical trials, sofosbuvir plus RBV, with or without PegIFN, has demonstrated SVR rates in excess of $80 \%$ for most cirrhotic patients and greater than or equal to $90 \%$ in the majority of treatment naïve patients. Based on these data, the AASLD/ IDSA guidance recommended sofosbuvir for the treatment of HCV patients. The combination of ledipasvir/sofosbuvir as a STR has been evaluated in large phase 3 clinical trials and demonstrated minimal toxicities and high efficacy, with an overall SVR of $97 \%$ in genotype 1 infected patients, without the need for either interferon or RBV. With recent FDA approval of ledipasvir/sofosbuvir, the majority of patients with chronic genotype $1 \mathrm{HCV}$ may achieve SVR in as short as 8 or 12 weeks of treatment with a once daily STR. Studies are underway to evaluate whether the duration of therapy may be further reduced by increasing the number of DAAs with different mechanisms of action in HCV regimens.

\section{Acknowledgements}

Copy editing and organizational advice was provided by the following people at Gilead Sciences, Foster City, California : Nika Burger, Diana Brainard, Betty Chiang, Bill Guyer, Bruce Kreter, Chris Lahart, Jamie Zagorski, Brent Peterson, and Anita Mathias; concept was developed by: George wu of the university of connecticut.

Table 4. Recommended treatment duration for once daily administered ledipasvir/sofosbuvir in patients with chronic HCV genotype 1

\begin{tabular}{ll}
\hline Patient population & Recommended treatment duration \\
\hline Treatment-naïve with or without cirrhosis & 12 weeks \\
$\begin{array}{l}\text { Treatment-naïve patients without cirrhosis who have } \\
\text { pretreatment HCV RNA less than } 6 \text { million IU/mL }\end{array}$ & Consider 8 weeks \\
Treatment-experienced* without cirrhosis & 12 weeks \\
Treatment-experienced* with cirrhosis & 24 weeks \\
\hline
\end{tabular}

${ }^{*}$ Treatment-experienced patients who have failed treatment with either PegIFN + ribavirin or an HCV protease inhibitor + PegIFN + ribavirin. ${ }^{20}$ 


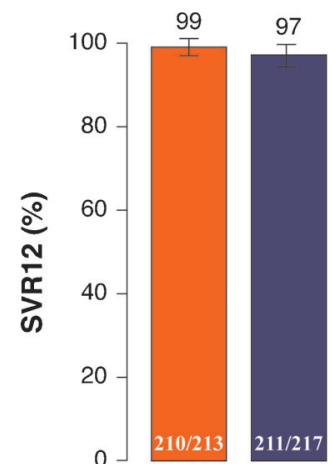

12 weeks

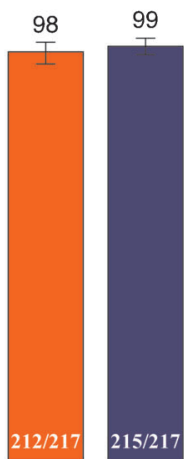

24 weeks

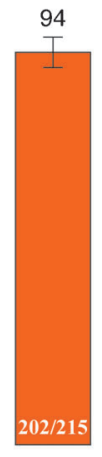

8 weeks

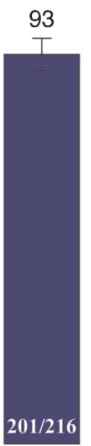

ION-3

GT 1 treatment-naive non-cirrhotics
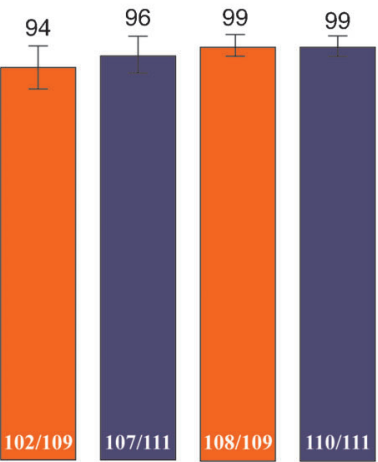

ION-1

GT 1 treatment-naive including cirrhotics

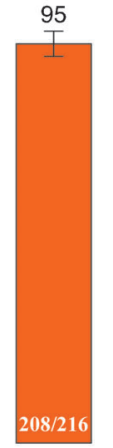

12 weeks
12 weeks

24 weeks

\section{ION-2}

GT 1 treatment-experienced including cirrhotics and $\mathrm{PI}$ failures

Fig. 5. ION phase 3 program (ION-1, ION-2, ION-3) efficacy summary. Error bars represent $95 \%$ confidence intervals.

\section{Conflict of interest}

None

\section{Author contributions}

Manuscript writing (TM, CS, SS).

\section{References}

[1] Chae HB, Park SM, Youn SJ. Direct-acting antivirals for the treatment of chronic hepatitis C: open issues and future perspectives. Scientific World Journal 2013;2013:704912. doi: 10.1155/2013/704912.

[2] Meanwell NA, Watkins WJ. Introduction to Hepatitis C Virus (HCV) Therapies Special Thematic Issue. J Med Chem 2014;57:1625-1626. doi: 10.1021 /jm5000936.

[3] Stedman C. Sofosbuvir, a NS5B polymerase inhibitor in the treatment of hepatitis C: a review of its clinical potential. Therap Adv Gastroenterol 2014; 7:131-140. doi: 10.1177/1756283X13515825.

[4] Gerber L, Welzel TM, Zeuzem S. New therapeutic strategies in HCV: polymerase inhibitors. Liver Int 2013;33(Suppl):85-92. doi: 10.1111 /liv.12068.

[5] Aghemo A, De Francesco R. New horizons in hepatitis C antiviral therapy with direct-acting antivirals. Hepatology 2013;58:428-438. doi: 10.1002 /hep.26371.

[6] Mosley RT, Edwards TE, Murakami E, Lam AM, Grice RL, Du J, et al. Structure of hepatitis $C$ virus polymerase in complex with primer-template RNA. J Virol 2012;86:6503-6511. doi: 10.1128/JVI.00386-12.

[7] Lam AM, Espiritu C, Bansal S, Micolochick Steuer HM, Niu C, Zennou V, et al. Genotype and subtype profiling of PSI-7977 as a nucleotide inhibitor of hepatitis C virus. Antimicrob Agents Chemother 2012;56:3359-3368. doi: 10.1128/AAC.00054-12.

[8] Sofia MJ, Bao D, Chang W, Du J, Nagarathnam D, Rachakonda S, et al. Discovery of a beta-d-2'-deoxy-2'-alpha-fluoro-2'-beta-C-methyluridine nucleotide prodrug (PSI-7977) for the treatment of hepatitis C virus. J Med Chem 2010;53:7202-7218. doi: 10.1021/jm100863x.

[9] De Clercq E. Dancing with chemical formulae of antivirals: A panoramic view (Part 2). Biochem pharmacol 2013;86:1397-1410. doi: 10.1016/j.bcp .2013.09.010.

[10] Gentile I, Borgia F, Buonomo AR, Castaldo G, Borgia G. A novel promising therapeutic option against hepatitis $C$ virus: an oral nucleotide NS5B polymerase inhibitor sofosbuvir. Curr Med Chem 2013;20:3733-3742. doi: $10.2174 / 09298673113209990178$.
[11] http://www.sovaldi.com/, accessed December 10, 2014.

[12] Kirby B, Gordi T, Symonds W, Mathias A. Population Pharmacokinetics of sofosbuvir and its major metabolite (GS-331007) in healthy and HCVinfected adult subjects. Hepatology 2013,58:746A-747A.

[13] Mathias A. Clinical Pharmacology Workshop in HIV (and HCV) PResentations on New DAA's oral HCV drugs in phase 3 development now. Proceedings 14th International Workshop on Clinical Pharmacology of HIV Thearpy, 2013; Amsterdam, Netherlands.

[14] Rodriguez-Torres M. Sofosbuvir (GS-7977), a pan-genotype, direct-acting antiviral for hepatitis C virus infection. Expert Rev Anti Infect Ther 2013;11: 1269-1279. doi: 10.1586/14787210.2013.855126.

[15] Lawitz EJ. The effect of Hepatic Impairment on the Pharmacokinetics and anti-viral activity of PSI-7977 in Hepatitis C infected subjects treated for seven days. European Association for the Study of Liver Disease, 2012; Barcelona, Spain.

[16] Cornpropst M, Denning J, Clemons D, Marbury T, Alcorn H. The Effect of Rena Impairment and End Stage Renal Disease on The Single-Dose pharmacokinetics of PSI-7977. J Hepatol 2012;56:S433. doi: 10.1016/S0168-8278 (12)61113-1.

[17] Cornpropst M. PSI-7977 Has no effect on QTcF intervals at Therapeutic or Supratherapeutic Doses. 62nd Annual Meeting of the American Association for the Study of Liver Diseases (AASLD). 2011; San Francisco, CA, USA

[18] Lawitz E, Gane EJ. Sofosbuvir for previously untreated chronic hepatitis C infection. N Engl J Med 2013;369:678-679. doi: 10.1056/NEJMc1307641.

[19] Osinusi A, Meissner EG, Lee Y], Bon D, Heytens L, Nelson A, et al. Sofosbuvir and ribavirin for hepatitis $C$ genotype 1 in patients with unfavorable treatment characteristics: a randomized clinical trial. JAMA 2013;310:804811. doi: 10.1001/jama.2013.109309.

[20] Jacobson IM, Gordon SC, Kowdley KV, Yoshida EM, Rodriguez-Torres M, Sulkowski MS, et al. Sofosbuvir for Hepatitis C Genotype 2 or 3 in Patients without Treatment Options. N Engl J Med 2013;368:1867-1877. doi: 10.1056/NEJMoa1214854.

[21] Zeuzem S, Dusheiko GM, Salupere R, Mangia A, Flisiak R, Hyland RH, et al. Sofosbuvir and ribavirin in HCV genotypes 2 and 3. N Engl J Med 2014;370: 1993-2001. doi: 10.1056/NEJMoa1316145.

[22] Sulkowski MS, Naggie S, Lalezari J, Fessel WJ, Mounzer K, Shuhart M, et al. Sofosbuvir and ribavirin for hepatitis $C$ in patients with HIV coinfection. JAMA 2014;312:353-361. doi: 10.1001/jama.2014.7734.

[23] Rodriguez-Torres M, Rodriguez-Orengo J, Gaggar A, Shen G, Symonds B, McHutchison J, et al. Sofosbuvir and Peginterferon alfa-2a/Ribavirin Treatment of naive Genotype 1-4 infected patients that are co-infected with HIV. Conference Reports for NATAP, ID Week, 2013; San Francisco, CA, USA.

[24] http://www.harvoni.com/, accessed December 10, 2014.

[25] Afdhal N, Reddy KR, Nelson DR, , Lawitz E, Gordon SC, Schiff E, et al. Ledipasvir and sofosbuvir for previously treated HCV genotype 1 infection. N Engl J Med 2014;370:1483-1493. doi: 10.1056/NEJMoa1316366. 
McQuaid T. et al: Sofosbuvir in HCV treatment

[26] Afdhal N, Zeuzem S, Kwo P, Chojkier M, Gitlin N, Puoti M, et al. Ledipasvir and sofosbuvir for untreated HCV genotype 1 infection. N Engl J Med 2014;370: 1889-1898. doi: 10.1056/NEJMoa1402454.
[27] Kowdley KV, Gordon SC, Reddy KR, Rossaro L, Bernstein DE, Lawitz E, et al. Ledipasvir and sofosbuvir for 8 or 12 weeks for chronic HCV without cirrhosis. N Engl J Med 2014;370:1879-1888. doi: 10.1056/NEJMoa1402355. 